\title{
Reptiles de la cuenca del río Unión, Valle de El General, Costa Rica
}

\author{
Luis Diego Arias Campos \\ Universidad Nacional, Escuela de Ciencias Ambientales, CR. Apdo. 86-3000, diegoac33@hotmail.com \\ Recibido 12-IV-2016 • Corregido 06-V-2016 • Aceptado 30-VI-2016
}

\begin{abstract}
Reptiles of the Unión river basin, Valle de El General, Costa Rica. Reptiles are unappreciated, mainly for their appearance, yet they have important functions in ecosystems, such as population control and as food for other species. I made monthly surveys (June 2014 to June 2015) in 20 sectors (15X200 m each) on both sides of Unión river in southern Costa Rica, along $12 \mathrm{~km}$ transects. I found 24 species of reptiles; one introduced, one endemic and three that range between Nicaragua and Panama; by group: nine snakes, one turtle, and 12 lizard iguana. Some, especially snakes, are killed by the settlers, even though most are not poisonous. This knowledge will be important in the management and conservation of watersheds in southern Costa Rica.
\end{abstract}

Key words: snake, turtle, iguana, lizard, watershed, conservation.
RESUMEN: Los reptiles son poco apreciados, principalmente por su aspecto. Sin embargo poseen funciones importantes en los ecosistemas, como control de poblaciones y como alimento para otras especies. Realicé recorridos mensuales de junio de 2014 a junio de 2015 en 20 sectores de $15 \times 200$ m en ambas orillas del río Unión, sur de Costa Rica, a lo largo de $12 \mathrm{~km}$. Encontré 24 especies de reptiles: una introducida, una endémica y tres con distribución entre Nicaragua y Panamá; por grupo, nueve de serpientes, una de tortuga, 12 de lagartija y una de iguana. Algunas, especialmente las serpientes, son sacrificadas por los pobladores, a pesar de que la mayoría no son venenosas. Su conocimiento será de importancia en el manejo y la conservación de las cuencas hidrográficas del sur de Costa Rica.

Palabras clave: serpiente, tortuga, iguana, lagartija, cuenca hidrográfica, conservación.
La clase Reptilia se encuentra representada en Costa Rica por 239 especies, lista que se espera que siga en aumento (Bolaños, Savage \& Cháves, 2011). Son de sangre fría, con presencia o ausencia de patas. Es común encontrarlos en rocas, troncos, escombros y otros sitios. Se diferencian de los anfibios por tener piel más seca y una menor dependencia de humedad (Leenders, 2001).

Entre los grupos de reptiles presentes en Costa Rica se encuentran los lagartos, las lagartijas, las tortugas, las iguanas o garrobos y las serpientes (Savage, 2002). Algunas especies son despreciadas por los humanos, debido al aspecto de algunas especies o por ser muy venenosas; ya que algunas poseen toxinas letales (Solórzano, 2001).

En Costa Rica se conocen 22 de las especies de serpientes venenosas. Este grupo -al igual que otros reptiles- se han visto amenazados por la perdida de hábitat, contaminación, y matanza por desprecio o miedo a ser mordido. Otro riesgo que corren es la extracción ilegal para domesticación; algunas especies por sus particularidades naturales no logran sobrevivir en otro hábitat, lo que causa reducciones de poblaciones silvestres (Solórzano, 2001).

Esta lista tiene como objetivo dar a conocer las especies de reptiles más comunes que habitan la cuenca del rio Unión; estos datos son de importancia en la toma de decisiones para el manejo de esta cuenca hidrográfica, así como para su conservación y la de los bosques ribereños.

El Valle de El General posee un clima húmedo tropical con tres a cuatro meses de estación seca. Gran parte de esta zona ha sido utilizada para actividades agropecuarias expansivas que ha llevado a la pérdida de hábitat de algunas especies silvestres. Sumado a esto, la contaminación, la tala ilegal y la cacería, han reducido algunas poblaciones a tal punto que actualmente solo es posible encontrarlas en las áreas protegidas al sur del país (Janzen, 1991; Leenders, 2001). Las orillas de este río son 
utilizadas principalmente para pastoreo, cultivo de café, caña y otros.

Realicé recorridos mensuales desde junio de 2014 a junio de 2015 en 20 sectores de 15X200 m a lo largo de $12 \mathrm{~km}$; desde la confluencia de la Quebrada Pavón y el Río San Rafael, coordenadas $9^{\circ} 19^{\prime} 13^{\prime \prime} \mathrm{N}, 83^{\circ} 31^{\prime} 01^{\prime \prime} \mathrm{W}$, a
867 msnm, hasta la confluencia entre el Río Unión y el Río General, coordenadas 9 $9^{\circ} 14^{\prime} 04.7^{\prime \prime} \mathrm{N}, 83^{\circ} 34^{\prime} 06.0^{\prime \prime} \mathrm{W}$, a $540 \mathrm{msnm}$.

En el cuadro 1 se muestran la especie, el nombre común y el origen (nativa, introducida o endémica) de las especies que habitan en el rio Unión.

CUADRO 1

Reptiles de la cuenca del río Unión.

Especie
Familia Boidae
Boa constrictor Linnaeus, 1758
Familia Colubridae
Clelia clelia (Daudin, 1803)
Lampropeltis triangulum (Lacépede, 1789)
Oxybelis fulgidus (Daudin 1803)
Phrynonax poecilonotus (Günther, 1858)

Familia Corytophanidae

Basiliscus basiliscus (Linnaeus, 1758)

Basiliscus plumifrons Cope, 1876

Corytophanes cristatus (Merrem, 1820)

\section{Familia Crotalinae}

Porthidium volcanicum Solorzano, 1995 (?)

\section{Familia Dactyloidae}

Anolis polylepis Peters, 1874

Anolis sp.

\section{Familia Diploglossidae}

Diploglossus bilobatus (O'shaughnessy, 1874)

\section{Familia Dipsadidae}

Imantodes sp.

Rhadinaea decorata (Günther, 1858)

Xenodon rabdocephalus (Wied-Neuwied, 1824)

\section{Familia Iguanidae}

Iguana iguana (Linnaeus, 1758)

\section{Familia Kinosternidae}

Kinosternon scorpioides (Linnaeus, 1766)

\section{Familia Phrynosomatidae}

Sceloporus malachiticus Cope, 1864

\section{Familia Polychrotidae}

Polychrus gutturosus Berthold, 1846

\section{Familia Scincidae}

Scincella cherriei (Cope, 1893)

\section{Familia Sphaerodactylidae}

Gonatodes albogularis (Duméril \& Bibron, 1836)

\section{Familia Teiidae}

Holcosus festivus (Lichtenstein, 1856)

Holcosus quadrilineatus (Hallowell, 1861)

amilia Viperidae

Nombre común

Origen

\begin{tabular}{|c|c|}
\hline Bécquer & Nativa \\
\hline Zopilota & Nativa \\
\hline Coral falsa & Nativa \\
\hline Bejuquilla verde & Nativa \\
\hline Pajarera & Nativa \\
\hline Basilisco & Nativa \\
\hline Basilisco & Nativa \\
\hline Perrozompopo & Nativa \\
\hline Toboba costarricense & Endémica \\
\hline Lagartija & Nativa (CR-Pan) \\
\hline Lagartija & Nativa \\
\hline Lagartija caimán & Nativa (Nic-Pan) \\
\hline Bejuquilla café & Nativa \\
\hline Espanta vieja & Nativa \\
\hline Falsa terciopelo & Nativa \\
\hline Iguana & Nativa \\
\hline Tortuga & Nativa \\
\hline Lagartija espinosa & Nativa \\
\hline Camaleón & Nativa \\
\hline Esquinco de bosque & Nativa \\
\hline Gecko & Introducida \\
\hline Chisbala & Nativa \\
\hline Chisbala & Nativa (Nic-Pan) \\
\hline Terciopelo & Nativa \\
\hline
\end{tabular}


En total se muestran 24 especies -dos de ellas identificadas a nivel de género-, las cuales se clasifican en 15 familias. La familia con más especies fue Colubridae, con cuatro, mientras que las familias Boidae, Crotalinae, Dipoglossidae, Iguanidae, Kinosternidae, Phrynosomatidae, Polychrotidae, Scincidae, Sphaerodactylidae y Viperidae, fueron representadas por una especie. Se encontraron nueve especies de serpientes, una especie de tortuga, 12 especies de lagartijas y una especie de iguana.

Para el caso de las serpientes, de las nueve encontradas, dos (B. asper y P. volcanicum) son venenosas. El veneno de $B$. asper es utilizado para fabricar antivenenos contra mordeduras en humanos y animales. Algunas especies no venenosas como C. clelia y L. triangulum poseen una importante función como controladores de poblaciones de algunas especies silvestres, incluyendo serpientes venenosas (Solórzano, 2001).

La especie $P$. volcanicum se incluye con duda debido a que solo se observó un individuo cerca del río General, el cual huyó y no pudo observarse mejor. Era similar a $B$. asper, la cual según la descripción de Solórzano (2001) los patrones de coloración de $B$. asper son triangulares, no así en el individuo observado.

Algunas especies de serpientes -inclusive las no venenosas- son sacrificadas por humanos. En ocasiones este comportamiento se da por desconocimiento de la especie, o simplemente por el temor que causan estos animales. La cacería de la especie I. iguana, ha sido una práctica común en zona.

Es probable que en esta lista hayan quedado excluidas otras especies como las arborícolas, así como las nocturnas que pueden habitar en esta zona, las cuales requieren de otros métodos de muestreo y estudios más exhaustivos. Sin embargo este trabajo se muestra como base para el conocimiento de la biodiversidad de la zona y para futuras investigaciones.

Las actividades humanas han provocado el desplazamiento y reducción de especies en el sur de Costa Rica. Este es apenas uno de los primeros intentos de conocer la biodiversidad de la cuenca del Río Unión, con el fin de promover el adecuado manejo y la conservación de las cuencas hidrográficas y sus ecosistemas en el Valle de El General.

\section{AGRADECIMIENTOS}

A Oscar Arias Segura por su apoyo en los nombres comunes. A Carmen Campos Valverde y Johan Barboza Tames por el apoyo en el trabajo de campo. A Gustavo Vargas Rojas y Juan Pablo Villegas Espinoza por sus recomendaciones en la preparación de este manuscrito.

\section{REFERENCIAS}

Bolaños, F., Savage, J. \& Chaves, G. (2011). Anfibios y Reptiles de Costa Rica. Listas Zoológicas Actualizadas UCR:

http://museo.biologia.ucr.ac.cr/Listas/LZAPublicaciones.htm.

Janzen, D. (ed.). (1991). Historia natural de Costa Rica. San Pedro: Editorial Universidad de Costa Rica.

Leenders, T. (2001). A guide to Amphibians and Reptiles of Costa Rica. United States: A Zona Tropical Publication.

Savage, J. (2002). The Amphibians and Reptiles of Costa Rica: A Herpetofauna between Two Continents, between Two Seas. Chicago: University of Chicago Press.

Solórzano, A. (2001). Serpientes de Costa Rica. Heredia: Editorial INBio. 
